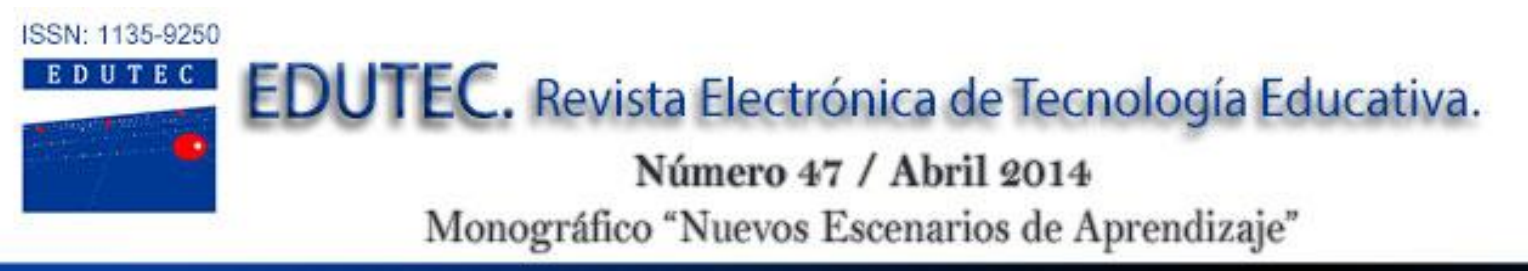

\title{
DISEÑO DE AUTOESTUDIOS MULTIMEDIA PARA COMPETENCIAS DIGITALES: CASO DEL PRIMER MOOC LATINOAMERICANO
}

\author{
HOW TO DESIGN MULTIMEDIA INSTRUCTION TO TEACH DIGITAL COMPETENCIES: \\ THE CASE OF THE FIRST MOOC IN LATIN AMERICA
}

Maria de Lourdes Gomez Porras; I gomez_p@yahoo.com

Rosario Celaya Ramirez; rosario.celaya@tecvirtual.mx

Maria Soledad Ramirez Montoya; solramirez@tecvirtual.mx

Universidad Virtual, Tecnológico de Monterrey.

\begin{abstract}
RESUMEN
Esta investigación analiza los elementos de diseño de ocho autoestudios multimedia (AM) que ayudaron a desarrollar dos competencias digitales de Alfabetización Informativa (AI) durante el primer seminario MOOC en Latinoamérica. El análisis se realizó desde dos perspectivas: principios de la Teoría Cognitiva del Aprendizaje Multimedia (TCAM) y la forma de incorporar los AM al seminario. Se utilizó el método cualitativo de estudio de caso. Mediante el análisis de documentos, entrevistas y observación se estudiaron dos categorías: desarrollo de competencias y elementos de los AM. Se encontraron cuatro elementos que favorecen el desarrollo de competencias, dos relacionados con la TCAM y dos con la integración de los AM al seminario: principio de coherencia, principio de personalización, conectivismo y aprendizaje activo.
\end{abstract}

Palabras clave: Multimedia, competencias digitales, alfabetización informativa, MOOC, conectivismo, aprendizaje activo, diseño instruccional, Teoría Cognitiva del Aprendizaje Multimedia.

\section{ABSTRACT}

The aim of this paper is to identify the instructional design elements in eight multimedia materials that prompted the development of Informational literacy competencies during a MOOC. Multimedia material is analyzed from two perspectives: the principles of the Cognitive Theory of Multimedia Learning (CTML), and the instructional design applied in the seminary. The qualitative research was conducted through a case study methodology, applying documents analysis, interviews and observation, two constructs were studied: development of digital competencies, and elements of instructional design in multimedia. Findings showed that two principles from the CTML were relevant, coherence and personalization; and two others derived from the design of the MOOC: connectivism and active learning.

Keywords: Multimedia, digital competencies, informational literacy, MOOC, connectivism, active learning, learning by doing, instructional design, Cognitive Theory of Multimedia Learning. 


\section{INTRODUCCIÓN}

Las competencias digitales de la alfabetización informativa (AI) son un factor clave para el desarrollo personal y social. La UNESCO (2003) las considera un requisito fundamental para el derecho humano de la educación a lo largo de la vida. Hoy en día la alfabetizado no basta para participar activamente en la sociedad, se requiere la multialfabetizado (Licea, 2009), de la cual forma parte la Al.

Por tales motivos, es crítico conocer las mejores formas de enseñar estas competencias. Especialmente en Latinoamérica, donde se requiere encontrar fórmulas para capacitar eficaz y efectivamente a grandes grupos de docentes que a su vez ayuden a sus alumnos a desarrollar sus competencias digitales.

En este contexto surgió el seminario objeto de este estudio de caso: Formación de educadores en ambientes a distancia para el desarrollo de competencias digitales en el uso de REA (recursos educativos abiertos). Seminario impartido utilizando un entorno de aprendizaje innovador: la educación masiva en línea combinada con el uso los recursos educativos abiertos. El seminario se convirtió en el primer curso masivo abierto en línea (MOOC, por sus siglas en inglés) en Latinoamérica.

El seminario fue organizado por SINED-CLARISE, después de que este grupo de universidades mexicanas, miembros de la Comunidad Latinoamericana Abierta Regional de Investigación Social y Educativa (CLARISE), ganara la convocatoria del Sistema Nacional de Educación a Distancia (SINED) en México para desarrollar un proyecto de educación a distancia para el período 2012-2013. La coordinación estuvo a cargo de investigadores de una universidad mexicana con presencia nacional e internacional a través de su universidad virtual.

El proyecto SINED-CLARISE para la educación a distancia se sustentó en el método de investigación-acción, donde se trabajó con el tema del movimiento educativo abierto para ambientes a distancia con enfoque en la formación en competencias (Ramírez, 2013).

El objetivo del seminario fue ayudar a los docentes a desarrollar competencias digitales y de diseño instruccional para integrar REA en los ambientes de educación a distancia. Estas competencias se basan en el desarrollo de las competencias digitales de la Al.

Una de las características principales del curso fue la incorporación de autoestudios multimedia (AM) en forma de videos, grabados especialmente por expertos en los temas del seminario. El contenido de los videos fue predefinido bajo un plan estratégico, y complementaba diversas actividades del seminario. A partir de este contexto y con la intención de contribuir a la mejora de las prácticas de enseñanza de las competencias digitales de la Al en los cursos masivos y través del uso de AM, surgió la pregunta de investigación: ¿Cuáles son los elementos del diseño de los AM que ayudan a desarrollar competencias digitales para que se cumplan los objetivos de Al en un seminario MOOC de educación continua? 
La investigación estudió dos competencias digitales: el acceso a la información en forma efectiva y eficiente, y la evaluación de la información de forma crítica y competente. Se consideraron los elementos de diseño instruccional desde dos puntos de vista: (1) las características de los mismos materiales instruccionales, basando el análisis en los principios de la Teoría Cognitiva del Aprendizaje Multimedia (TCAM) y (2) la perspectiva de cómo se alinean los videos con el diseño integral del seminario.

\section{COMPETENCIAS DIGITALES DE LA ALFABETIZACIÓN INFORMATIVA (AI)}

La alfabetización informativa (Al) es una parte de la alfabetización digital. La alfabetización digital incluye aspectos técnicos, habilidades y competencias para el uso de herramientas tecnológicas (Eshet-Alkalai, 2004). Y la Al se enfoca en las competencias digitales que permiten identificar cuando se necesita información, cómo localizarla, evaluarla y usarla (ACRL, 2000; UNESCO, 2003; IFLA, 2005). A partir de esa definición, se han generado estándares para facilitar la creación de programas educativos. Este estudio se fundamenta en la propuesta de la IFLA (Lau, 2006) que agrupa las competencias digitales en: acceso, evaluación y uso de la información. Esta clasificación permite centrarse en las partes esenciales del proceso. De estas competencias, se estudiaron las dos primeras: acceso a la información y la evaluación de la información.

La competencia de acceso a la información, abarca dos aspectos. El primero es definir la necesidad de la información, esto implica entender la situación y el contexto donde surge la necesidad, identificar qué se requiere, e iniciar la búsqueda. El segundo es localizar la información, esto requiere identificar y evaluar posibles fuentes de información, desarrollar estrategias de búsqueda, realizar la búsqueda y obtener de la información deseada.

La competencia de evaluación de la información, abarca también dos aspectos. El primero es la valoración de la información, que incluye: análisis, generalización e interpretación, selección de lo relevante, síntesis y evaluación (exactitud y relevancia). El segundo aspecto, es la organización de la información, que incluye: crear categorías (esquemas o diagramas), organizar (sistema de almacenamiento y referencias) y determinar la información más valiosa y útil.

Diversos autores señalan que la enseñanza de las competencias de alfabetización digital debe (1) mostrar el proceso integral donde surgen las competencias (Keene, Colvin y Sissons, 2010), (2) basarse en la complejidad cognitiva de la competencia (ACRL, 2000; Keene y otros, 2010), y (3) aplicar estrategias como el aprendizaje activo (Lau, 2006; Oakleaf y VanScoy, 2010; Dunaway, 2011).

Transue (2013) y Dunaway (2011) han señalado que el conectivismo es un paradigma adecuado para la enseñanza de las competencias digitales. Los MOOC basados en este paradigma (Siemens, 2005) son innovadores al crear redes de conocimiento distribuidos. El aprendizaje y el 
conocimiento se basa en la diversidad de opiniones, es un proceso de conexión de fuentes de información especializadas que puede residir en herramientas (Siemens, 2005).

\section{DISEÑO DE AUTOESTUDIOS MULTIMEDIA (AM)}

Para determinar los elementos de los autoestudios multimedia (AM) se revisaron las teorías relacionadas con el aprendizaje basado en multimedia. Sweller (2005) propone la teoría de la carga cognitiva (TCC) y Mayer (2005) la teoría cognitiva del aprendizaje multimedia (TCAM). Ambas teorías se basan en la suposición de que la capacidad cognitiva en la memoria operativa es limitada. En consecuencia, sugieren usar estrategias de diseño instruccional que optimice el uso de la memoria operativa y eviten una sobre carga cognitiva (de Jong, 2009).

Las cargas cognitivas relacionadas con el uso de material multimedia pueden ser de tres tipos: (1) carga cognitiva intrínseca, es la carga inherente al contenido a ser aprendido, (2) carga cognitiva extrínseca, es la carga ocasionada por la forma en que se presenta el contenido, y (3) la carga relevante, que es la carga cognitiva que impone el mismo proceso de aprendizaje (de Jong, 2009; Sweller, 2005).

La TCAM establece diez principios de diseño que pretenden mejorar el aprendizaje (Mayer, 2008):

- Principios que ayudan en la reducción de la carga extrínseca: coherencia, señalización, contigüidad espacial, contigüidad temporal y redundancia.

- Principios que ayudan a manejar los procesos esenciales e intrínsecos: pre-entrenamiento, segmentación y modalidad.

- Principios que facilitan los procesos relevantes: multimedia y personalización.

A pesar de que la TCAM y la TCC se basan en evidencia empírica, han recibido críticas importantes. Astleitner y Wiesner (2004) señalan que se ignoran los factores individuales, como la motivación o los estilos de aprendizaje que afectan directamente la forma en que los usuarios usan los materiales multimedia y las expectativas que se crean de los mismos.

A su vez, de Jong (2010) señala que muchos de los estudios se han llevado a cabo en condiciones de laboratorio con participantes reclutados para los experimentos, por lo que no tenían ningún interés en los contenidos de los multimedia.

\section{METODOLOGÍA}

Se utilizó el método cualitativo de estudio de caso para analizar las dos categorías: desarrollo de competencias y elementos de diseño de AM. Cada una con diversos indicadores (ver Figura 1). 


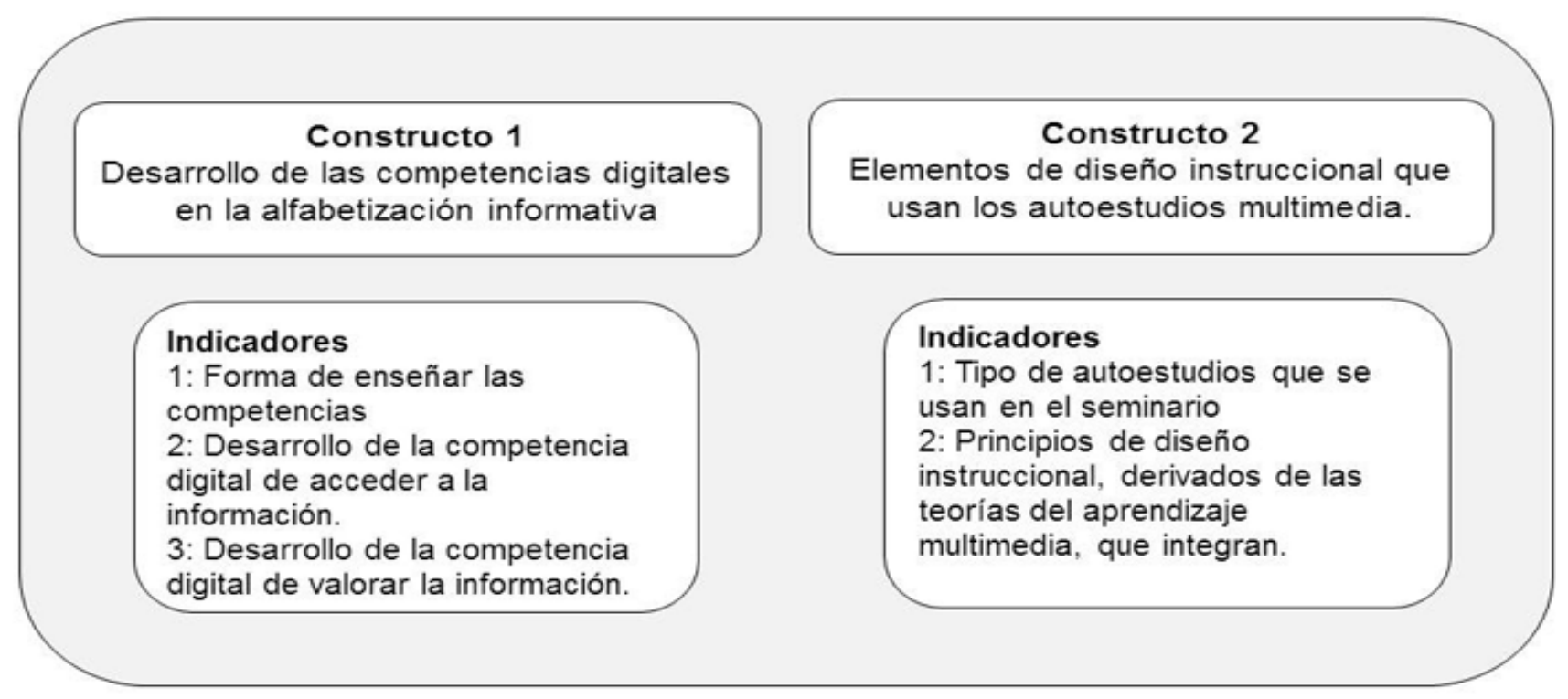

Figura 1. Categorías e indicadores (fuente: elaboración por la autora).

Se usaron tres técnicas de recolección: análisis de documentos, observación no participativa y entrevistas (con enfoque - preguntas abiertas con guía- y tradicionales - encuestas). Se empleo el cuadro de triple entrada para guiar el diseño de cada instrumento, la selección de las fuentes de información y para apoyar en la triangulación de las fuentes (la Figura 2 presenta una parte de este cuadro). 


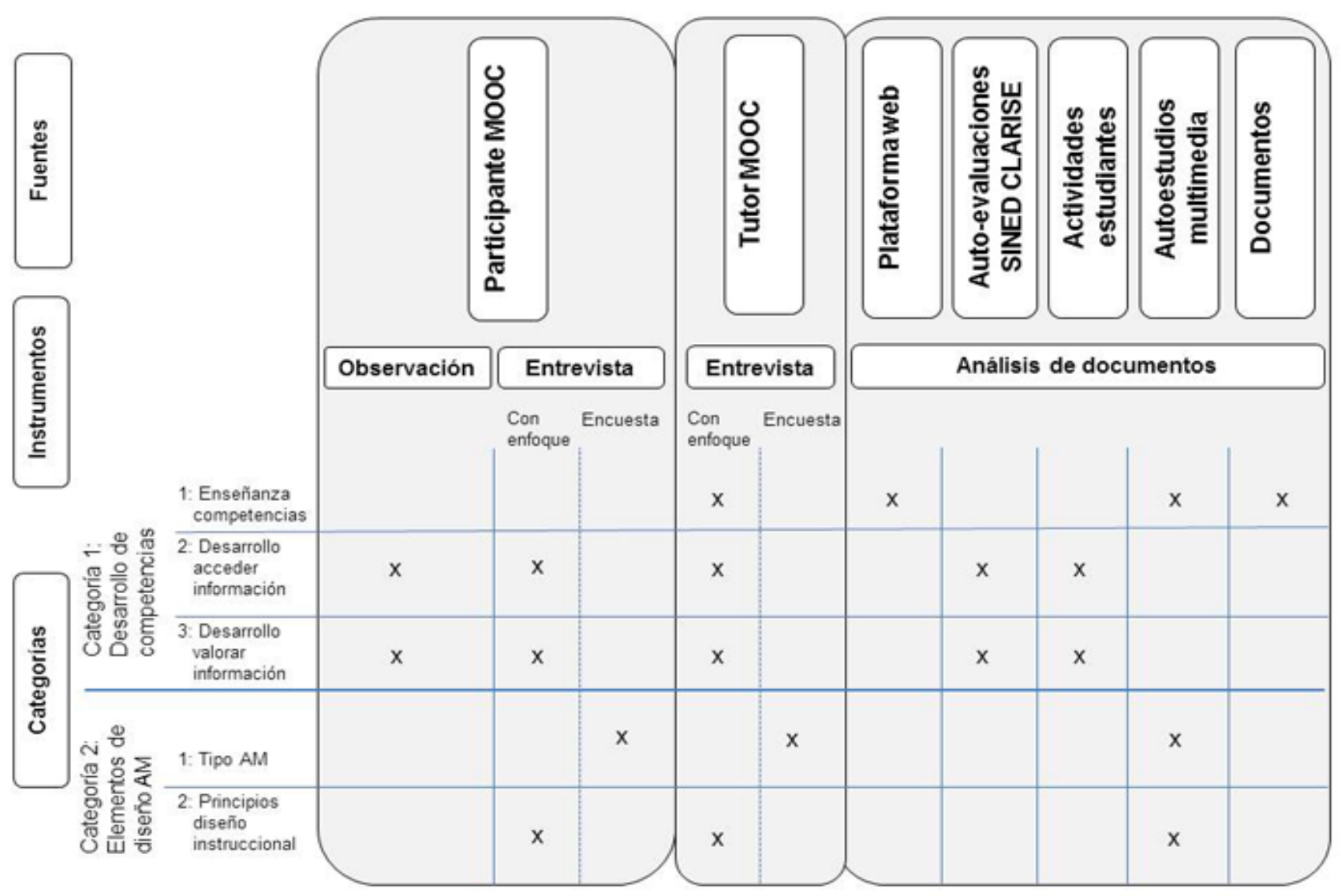

Figura 2. Relación fuentes, instrumentos, categorías e indicadores (fuente: elaboración por la autora).

El análisis de documentos fue aplicado en:

1) Documentos de los organizadores (minutas y presentaciones) para conocer cómo se definieron las estrategias educativas. Se analizó la definición del (1) enfoque del seminario, (2) contenido (básico/consulta, local/distribuido, propio/de terceros), (3) actividades (coevaluación, autoevaluación, aprender en red) y (4) AM para recoger datos relacionados con estrategias educativas y diseño (conectivismo, competencias como proceso integral).

2) Plataforma web del seminario para analizar el diseño del seminario y el material que enseñaba las competencias. Se analizó la organización del curso, contenido de cada módulo, contenido externo y AM, descripción de actividades (tareas y coevaluación) para recoger datos relacionados con estrategias educativas y diseño (conectivismo, competencias como proceso integral).

3) Autoevaluaciones diseñadas y aplicadas por el equipo SINED-CLARISE a los alumnos (diagnósticas, intermedias y finales) para para conocer el estado de las competencias en distintos momentos. Se recopilaron datos de las dos competencias: definición de la necesidad de información, estrategias de búsquedas, valoración de material (confiabilidad, credibilidad, calidad) y fuentes, y organización de información. 
4) Ocho AM para identificar cómo enseñaban las competencias digitales. Se recogieron datos (1) relacionados con las estrategias de aprendizaje, el diseño y forma de presentar las competencias y (2) de aplicar cada uno de los principios de TCAM.

5) Actividades de los alumnos (tarea y coevaluación) para encontrar evidencia de la aplicación y desarrollo de las competencias. Se recopilaron datos relacionados con la definición de la necesidad, búsquedas, valoración y organización de la información.

El instrumento consistió en un registro donde se incluían datos generales del documento analizado (fecha, título, ubicación, etc.) y específicos (descripción de datos, resumen y categorías e indicadores relacionados).

La observación se aplicó en los participantes del seminario en los foros, para identificar la aplicación y el desarrollo de competencias. En la observación cualitativa se trabaja con “episodios de relación única para formar una historia o una descripción única del caso" (Stake, 1999, p.62). Por tanto, los datos recogidos fueron comentarios que reflejaban la aplicación de las competencias. El instrumento usado fue una rejilla de observación que recababa datos generales (fecha, ubicación, datos del participante) y específicos (transcripción, contexto y categorías e indicadores relacionados).

Las entrevistas con enfoque a los alumnos y tutores tuvieron los objetivos de: (1) encontrar evidencias del desarrollo de las competencias en los participantes y del impacto de los AM y (2) conocer su opinión de las características de los AM. Se recogieron datos sobre (1) la forma en que los participantes definían, localizaban, valoraban y organizaban la información, y cómo afectaron (o no) los AM estos procesos, y (2) opinión sobre características de los AM relacionadas con los principios de TCAM.

Estas entrevistas consistieron de dos partes: (1) cómo aplicaban las competencias digitales los participantes y el impacto de los AM en ellas, y (2) las características de los AM. Las características estaban relacionadas con los principios de diseño de la TCAM. Sin embargo, no se utilizó el nombre del principio, sino una descripción del mismo. Por ejemplo, se preguntó por el uso de recursos no esenciales en los AM (música, diseño, sonido) y no por el principio de coherencia. A los tutores adicionalmente se les preguntó cómo enseñaban las competencias digitales los AM.

El instrumento de estas entrevistas consistió de dos partes, (1) una guía con aspectos generales para la entrevista y las diez preguntas que servirían como base, cinco sobre las competencias y cinco sobre las características de los AM, y (2) un formato para anotar las respuestas clasificándolas dependiendo del categoría e indicador con el que se relacionaban los datos. Stake (1999) señala que en la entrevista lo importante es entender lo que el sujeto entrevistado quiere decir, por tanto en este estudio el investigador usó preguntas de refuerzo y resúmenes para confirmar lo dicho por el sujeto. Esto permitió reconstruir la narración durante el análisis y dar la interpretación correcta. 
Para determinar el número de actividades a analizar, y de entrevistas y en cuestas, se usó el muestreo aleatorio e intencional. Aleatorio para obtener un panorama general del desarrollo de las competencias. Basándose en el principio del punto de saturación se analizaron 20 tareas y 20 coevaluaciones. Intencional para profundizar en el desarrollo de las competencias y el impacto de los AM en los participantes, a través de entrevistas semi-estructuradas y encuestas a dos tutores y cinco participantes. El resto de documentos (documentos organizadores, AM, sitio web, etc.) se analizaron en su totalidad.

Una vez recabados los datos se utilizó la triangulación metodológica, los datos de las diversas fuentes e instrumentos se organizaron de acuerdo con la categoría e indicador con los que estaban relacionados. Por ejemplo, los datos del desarrollo de la competencia de acceder a la información se agruparon. Se usó la suma categórica (Stake, 2010), identificando patrones, encontrando similitudes y estableciendo puntos en común para cada indicador y categoría. Así se identificaron tendencias y temas recurrentes, y empezó a surgir una imagen clara del caso. Usando la interpretación directa, también se identificaron ejemplos relevantes que ilustraban los fenómenos. De igual forma se usó la triangulación teórica con autores relevantes para cada categoría (TCAM y sus críticos, conectivismo, aprendizaje activo), lo que permitió una interpretación de los datos desde diversos ángulos.

Las preguntas de investigación, el marco teórico y los supuestos del estudio guiaron la interpretación y la organización de los hallazgos relacionando categorías e indicadores. De esta forma se le dio sentido a los datos y se empezaron a generar explicaciones del fenómeno estudiado.

\section{RESULTADOS}

Los resultados se presentan de acuerdo con las categorías e indicadores.

Desarrollo de las competencias. Esta categoría está formada por tres indicadores. En el primero, "forma de enseñar las competencias", se encontró que el seminario empleó el conectivismo. En palabras de los organizadores, el seminario se concibió como un experimento para enfocarse al conectivismo, se facilitó el aprendizaje en red, la interacción con otros, con herramientas y con contenido, que muchas veces "se encuentra distribuido (sitios web, blogs, redes sociales)". Este enfoque quedó reflejado en la metodología del seminario, el contenido y el diseño de las actividades (ver Figura 3). 


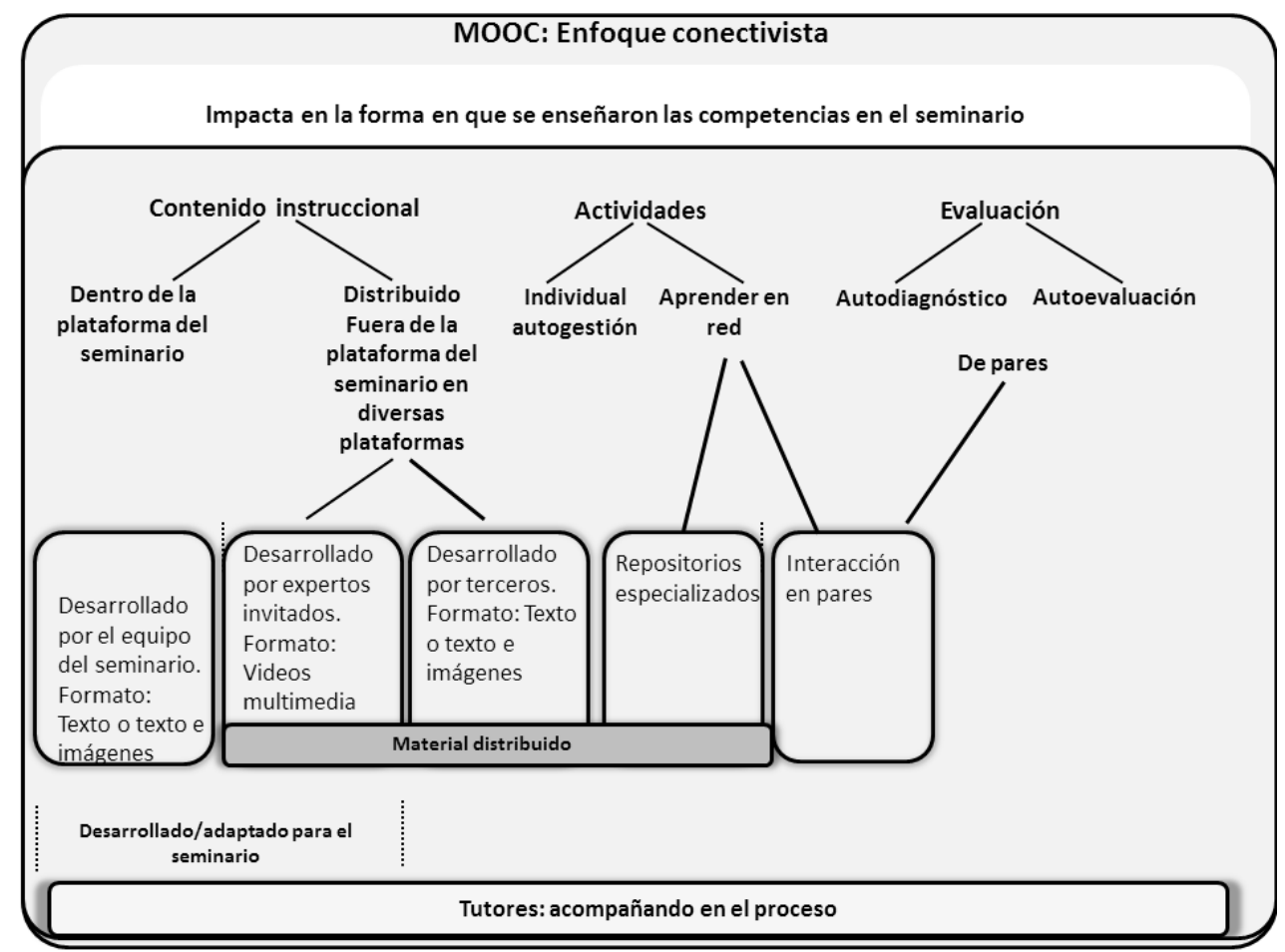

Figura 3. Enfoque conectivista (fuente: elaboración por la autora basado en el análisis de documentos).

El análisis de documentos y las entrevistas mostraron que los ocho AM eran videos de expertos que abordaban las dos competencias investigadas. Los videos fueron realizados basándose en parámetros predeterminados por los organizadores. Eran didácticos, claros y precisos. Mostraban contenido práctico y ejemplos de cómo buscar, valorar y usar información, los más relevantes se enfocaban al uso de bases de datos y repositorios especializados y formas de evaluar el contenido.

Las actividades del curso requerían la aplicación de las competencias y el uso de los contenidos (textos y AM). La tarea fue considerada como "adecuada" por los participantes, se debía definir la información requerida, crear una estrategia de búsqueda, realizar la búsqueda, analizar los resultados, interpretarlos, evaluar su calidad, organizarlos y definir su utilidad. Adicionalmente, la coevaluación fue considerada "útil", porque permitió aplicar la competencia de evaluar la información usando una rúbrica completa.

Los resultados del segundo indicador, "desarrollo de la competencia digital de acceder a la información", mostraron que para identificar la información requerida los participantes partían del objetivo de una actividad. Esta estrategia fue aplicada en las tareas y las coevaluaciones.

A pesar del desconocimiento inicial sobre motores especializados y repositorios académicos, de acuerdo con los entrevistados sus conocimientos aumentaron con el seminario. Estos temas se trataron en los videos y su aplicación fue requerida en las tareas, se halló que la mitad de los participantes usaron únicamente recursos provenientes de sitios académicos y especializados (el resto usaron una mezcla de sitios). 
En cuanto a la obtención de la información requerida, antes del seminario los participantes reportaron que no localizaban con rapidez recursos útiles que se ajustara a sus necesidades. Pero durante el seminario pusieron en práctica esta competencia. Esto se observo al analizar el material que incluyeron en sus tareas, porque fue adecuado, relevante y específico. Al final del curso la autoevaluación evidencio que los participantes que aún tenían dificultades para buscar recursos, lo asociaban con su limitada experiencia y conocimientos.

Los resultados del tercer indicador, "desarrollo de la competencia de evaluar la información", indicaron que la competencia se aplicó en las actividades. En las tareas, casi tres cuartas partes tenían información relevante y específica, lo que refleja que se hizo un análisis de la información. También, incluían textos concretos y claros que sintetizaban los recursos, así mismo la información se presentó de forma organizada y estructurada. Por su parte, en las coevaluaciones, en doce de veinte además de usar la rúbrica para dar retroalimentación se incluyeron comentarios detallados, que reflejaron un análisis profundo de la información evaluada.

Al inicio del curso la mitad de los participantes tenían un nivel avanzado o experto para determinar la credibilidad de la información. Al final, más de dos terceras partes expresaron no tener problemas para aplicar técnicas para evaluar la validez y veracidad de la información. La aplicación de esta competencia se aprecia en las tareas y las coevaluaciones. En las tareas, catorce de las veinte incluían información relevante y precisa. En las coevaluaciones los veinte alumnos aplicaron la competencia, aunque sólo la mitad incluyó comentarios detallados.

En cuanto a la forma de organizar información, los datos muestran una mejora entre el inicio y el final del seminario. Al inicio poco más de un cuarto reportó sentirse experto o con conocimiento avanzado para usar técnicas y métodos para organizar la información, durante el curso esto se modificó y al final más de tres cuartos señalaron sentirse sin ningún problema al organizar información obtenida por diversas fuentes.

Elementos de diseño de los AM. Esta categoría está formada por dos indicadores. En el primer indicador, "características de los AM", se encontró que todos los AM eran videos cortos presentados por expertos con contenido relevante y de calidad. La información se exponía en forma oral con apoyos visuales en texto y con pocos efectos adicionales.

En el segundo indicador, "principios derivados de la TCAM", los resultados muestran que los principios se aplicaron en forma variada y que parte de los efectos en los participantes dependieron de factores personales.

Los resultados por principio fueron:

- Coherencia. Todos los videos aplican el principio. Los participantes señalaron que los elementos "no distraían", "ni interferían" y permitían centrarse en el contenido.

- Señalización. Aplicado en partes muy breves en cinco de los videos. 
- Contigüidad espacial. No aplica.

- Contigüidad temporal. Aplicado en sólo un video.

- Redundancia. Aplicado en tres videos. Sin embargo, las entrevistas indicaron que no molestaba tener contenido duplicado (oral y escrita); al contrario, permitía centrarse en el contenido esencial al poder escoger si se leía o escuchaba la información.

- Preentrenamiento. Aplicado en todos los videos.

- Segmentación. No es posible ir a un segmento directamente pero sí adelantar, retrasar, detener o volver a repetir el video.

- Modalidad. Se aplica en el único video que utiliza la simulación. Sin embargo, se discutió ampliamente en las entrevistas. Se habló de la importancia de la música (auditivo) para ayudar al alumno a relejarse y concentrarse; de reforzar los estímulos visuales con "gráficos" para ayudar en la creación de mapas mentales. Dos entrevistadas, autodescritas como visuales, recalcaron la importancia de los esquemas, otro entrevistado, autodescrito como kinestésico, habló de la necesidad de usar imágenes atractivas para hacerlos más dinámicos y mantener la atención.

- Multimedia. Todos videos usan multimedia pero con pocos elementos gráficos (con excepción de texto).

- Personalización. La imagen general de los entrevistados es que el principio se aplicó, creando el efecto esperado, la percepción que el uso del lenguaje ayudó a crear un vínculo social. Una de las tutoras resaltó la importancia de que los expertos hablen directamente al espectador en forma clara, sencilla, precisa ya que esto los ayuda a acompañar al alumno para que se "sienta más seguro".

\section{DISCUSIÓN Y CONCLUSIONES}

En el presente apartado se da respuesta a la pregunta de investigación, se discuten los hallazgos y se presentan las conclusiones.

La pregunta de investigación planteada fue ¿cuáles son los elementos del diseño de autoestudios multimedia que ayudan a desarrollar competencias digitales para que se cumplan los objetivos de alfabetización informativa en un seminario MOOC de educación continua?

Con los resultados y la interpretación de los mismos, sustentada con el marco teórico, se estableció que hay dos grupos de elementos del diseño de autoestudios multimedia que ayudan a desarrollar competencias digitales: (1) los relacionados con los principios de diseño instruccional derivados de TCAM, en concreto el principio de coherencia y de personalización, y (2) los relacionados con el diseño instruccional usado en el seminario MOOC y aplicado en los autoestudios multimedia para integrarlos al mismo seminario, en concreto las estrategias del conectivismo y del aprendizaje activo. 
El principio de coherencia de la TCAM establece que el contenido no esencial debe ser reducido al mínimo para que los individuos puedan centrarse en la parte esencial del contenido. La aplicación de este principio en todos los videos facilitó el desarrollo de las competencias digitales al permitir que la atención de los participantes se centrase en el contenido relevante y práctico que enseñaban las competencias, y no en aspectos no esenciales, que "no distraían", como el diseño gráfico o la música. De acuerdo con Mayer (2008) el principio de coherencia ayuda a reducir la carga extrínseca (no esencial) en los procesos de aprendizaje, al evitar que se incluyan materiales que no aportan información relevante, de tal forma que toda la capacidad cognitiva se centra en los procesos centrales. De aquí se desprende que la aplicación de este principio es básica para ayudar a los participantes a enfocarse en los contenidos importantes.

El principio de personalización de TCAM establece que el uso de lenguaje informal ayuda a que los espectadores establezcan un vínculo con el expositor, y de esta forma se centren el procesamiento del contenido esencial (Mayer, 2008). La aplicación de este principio ayudó a que se creara un vínculo con el expositor y los participantes enfocaran su atención al contenido fundamental, es decir en el contenido que mostraba cómo buscar, evaluar o seleccionar información. Seis de los autoestudios multimedia aplicaron la personalización (aunque en distintos grados), lo cual dejó una imagen favorable en los entrevistados, quienes consideraron que el uso del lenguaje en los videos fue "ameno", de "adulto para adulto", con "terminología clara", "a la altura" al tiempo les transmitió calidez, se sintieron "más cerca de la persona". Esto implica que en un seminario MOOC, el uso de AM que usen expertos puede ayudar a crear una conexión personal con los participantes, bajando así las barreras y permitiendo que los procesos cognitivos ayuden a centrarse en el contenido principal.

Otros dos principios relevantes pero no determinantes son el de modalidad y el de redundancia, su impacto depende de los estilos de aprendizaje personales (auditivos, visuales o kinestésicos). El primero hace hincapié en utilizar los dos canales para procesar la información (visual y auditivo) y el segundo en evitar duplicar el texto (hablado y escrito). Aunque estos principios no se aplicaron en la mayoría de los autoestudios, el impacto en los participantes dependió de su forma de autodescribirse, ya sea como visuales (sugerían usar más gráficos y diagramas, pero veían bien que el texto oral se duplicara por escrito), auditivos (sugerían usar música, pero estaban de acuerdo en que todo el contenido se duplicara con texto oral) o kinestésicos (sugerían usar más recursos visuales y auditivos para hacerlos más dinámicos). Mayer (2008) establece que estos principios facilitan el procesamiento de la información al disminuir la carga cognitiva extrínseca, sin embargo Astleitner y Wiesner (2004) hacen notar que la TCMA ignora los factores individuales como los estilos de aprendizaje, que afectan el uso y las expectativas de los AM. Estos resultados ponen de manifiesto la importancia de estos factores personales.

En cuanto a los elementos de diseño instruccional usado en el seminario MOOC y aplicado para integrar los autoestudios, se encontró que el conectivismo y el aprendizaje activo ayudaban a desarrollar las competencias digitales de la Al. 
En cuanto al conectivismo como estrategia de enseñanza, el seminario MOOC utilizó estrategias conectivistas que impactaron la forma de enseñar las competencias en los AM. Los AM se diseñaron siguiendo un plan estratégico y de acuerdo con parámetros predefinidos, lo que hizo que su contenido fuera relevante para llevar a cabo la tarea y la coevaluación dentro del MOOC. Dunaway (2011) y Transue (2013) señalan que el uso del conectivismo para la enseñanza de las competencias digitales es adecuado ya que ambos sistemas tienen objetivos que están alineados. Transue (2013) agrega, "conceptos como pensamiento crítico, credibilidad, relevancia, validez, búsqueda de información, acceso a la información" son parte integral de ambos (p. 191).

En cuanto al uso del aprendizaje activo, los AM pueden desarrollar las competencias digitales cuando son parte de una estrategia de aprendizaje activo que incluye actividades para poner en práctica dichas competencias. El análisis de documentos y las entrevistas reflejaron que los AM explicaban cómo buscar, seleccionar y valorar la información, proporcionando información relevante y práctica que después era requerida en la tarea y coevaluación. La aplicación de las competencias en dichas actividades fue correcta y detallada (al definir la información requerida, seleccionar REA relevante después de una búsqueda y una valoración apropiadas, organizar los REA dentro de una actividad de aprendizaje, analizar y valorar las tareas de otros y determinar el contenido más útil). Cuando una persona participa en actividades aprende haciendo, este aprendizaje es esencial para el desarrollo de competencias digitales (Lau, 2006; Oakleaf y VanScoy, 2010). De aquí se desprende que la idoneidad de los AM para enseñar competencias en un contexto MOOC dependerá de la relevancia de su contenido y de la forma de integrarse con otras actividades del mismo curso.

Queda con este escrito una invitación a seguir analizando las posibilidades de los nuevos entornos de aprendizaje en experiencias de formación masiva. El realizar más investigaciones sobre la aplicación de los principios multimedia en cursos como el seminario MOOC, y no en condiciones de laboratorio, de tal forma que los participantes sean estudiantes interesados en un tema, y se puedan apreciar los factores que determinan la importancia de un principio. Con la intención de identificar: (a) concretamente qué principios son importantes en distintos contextos y por qué, (b) qué factores externos relacionados con el curso impactan sobre el valor de los principios, como tema del curso o duración, (c) qué otros factores individuales impactan, además de los estilos de aprendizaje, como la motivación o el conocimiento previo del tema, y (d) la forma en que los estilos de aprendizaje, o la percepción propia de éstos, impacta sobre la forma en que se valoran distintos principios.

\section{RECONOCIMIENTO:}

Este estudio se desarrolló en el marco del proyecto SINED-CLARISE para la educación a distancia (https://sites.google.com/site/sinedclarise/) apoyado por el Sistema Nacional de Educación a Distancia (SINED-cn-02/12), con la participación de los integrantes del proyecto. 


\section{REFERENCIAS}

Astleitner, H. y Wiesner, C. (2004). An integrated model of multimedia learning and motivation. Journal of Educational Multimedia and Hypermedia, 13, 3-21. Recuperado de http://www.editlib.org/p/5049

De Jong, T. (2010). Cognitive load theory, educational research, and instructional design: some food for thought. Instructional Science, 38(2), 105-134. Recuperado de http://doc.utwente.nl/83024/1/Jong10cognitive.pdf

Dunaway, M. K. (2011). Connectivism: Learning theory and pedagogical practice for networked information landscapes. Reference Services Review, 39(4), 675-685. DOI 10.1108/0090732111118668

Eshet-Alkalai, Y. (2004). Digital Literacy: A Conceptual Framework for Survival Skills in the Digital Era. Journal of Educational Multimedia and Hypermedia, 13(1), 93-106. Recuperado de http://www.openu.ac.il/Personal sites/download/Digital-literacy2004-JEMH.pdf

International Federation of Library Associations and Institutions (IFLA). (2005). The Alexandria Proclamation on information literacy and lifelong learning. Recuperado de http://archive.ifla.org/Ill/wsis/BeaconInfSoc.html.

Keene, J., Colvin, J., y Sissons J. (2010). Mapping Student Information Literacy Activity against Bloom's Taxonomy of Cognitive Skills. Journal of information literacy, 4(1), 6-21. Recuperado de http://ojs.lboro.ac.uk/ojs/index.php/JIL/article/view/PRA-V4-I1-2010$\underline{1 / 1451}$

Lau, J. (2006). Guidelines on information literacy for lifelong learning (final draft). International Federation of Library Associations and Institutions (IFLA). Recuperado de http://www.ifla.org/files/assets/information-literacy/publications/ifla-guidelines-en.pdf

Licea, J. (2009). La alfabetización informacional en el entorno hispanoamericano. Anales de Documentación, 12, 93-106. Recuperado de http://redalyc.uaemex.mx/pdf/635/63511932005.pdf

Mayer, R. (2005). Introduction to Multimedia Learning. En R. Mayer (Ed.), The Cambridge Handbook of Multimedia Learning (pp. 1-18). Nueva York, Estados Unidos: Cambridge University Press.

Mayer, R. (2008). Applying the science of learning: Evidence-based principles for the design of multimedia instruction. American Psychologist, 63(8), 760-769. DOI 10.1037/0003066X.63.8.760

Moreno, R. y Mayer, R. (2007). Interactive Multimodal Learning Environments. Educ Psychol Rev, 19, 309-326. DOI 10.1007/s10648-007-9047-2 
Oakleaf, M. y VanScoy, A. (2010). Instructional Strategies for Digital Reference: Methods to Facilitate Student Learning. Reference \& User Services Quarterly, 49(4), 380-390. Recuperado de: http://meganoakleaf.info/instructstratdigref.pdf

Ramírez, M. S. (2013). Retos y perspectivas en el movimiento educativo abierto de educación a distancia: estudio diagnóstico en un proyecto SINED. Revista de Universidad y Sociedad del Conocimiento (RUSC), 10(2), 170-186. Recuperado de: http://rusc.uoc.edu/ojs/index.php/rusc/article/view/v10n2-ramirez/v10n2-ramirez-es

Siemens, G. (2005). Connectivism: A learning theory for the digital age. International Journal of Instructional Technology and Distance Learning, 2(1). Recuperado de http://www.itdl.org/journal/jan 05/article01.htm

Stake, R. E. (1999). Investigación con estudio de casos. Madrid, España: Ediciones Morata. Recuperado de: http://es.scribd.com/doc/51397687/INVESTIGACION-CON-ESTUDIO-DECASOS-STAKE

Stake, R. E. (2010). Qualitative Research. Nueva York, Estados Unidos: The Guilford Press.

Sweller, J. (2005). Implications of Cognitive Load Theory for Multimedia Learning. En R. Mayer (Ed.), The Cambridge Handbook of Multimedia Learning (pp. 19-30). Nueva York, Estados Unidos: Cambridge University Press.

Transue, B.M. (2013). Connectivism and Information Literacy: Moving From Learning Theory to Pedagogical Practice. Public Services Quarterly, (9)3, 185-195. DOI: 10.1080/15228959.2013.815501

UNESCO. (2003). Prague Declaration on information literacy. Recuperado de http://portal.unesco.org/pv obj cache/pv obj id 0106E60518A5E4524F2E44C80D3B09C 654C80000/filename/PragueDeclaration.pdf

\section{Para citar este artículo:}

Gomez, M.L., Celaya, R. \& Ramirez, M.S. Diseño de autoestudios multimedia para competencias digitales: Caso del primer MOOC latinoamericano. EDUTEC, Revista Electrónica de Tecnología Educativa, 47.4 el $\quad$ Recuperado dd/mm/aa de http://edutec.rediris.es/Revelec2/Revelec47/n47 Gomez-Celaya-ramirez.html 